\title{
BMJ Open EduCan trial: study protocol for a randomised controlled trial on the effectiveness of pain neuroscience education after breast cancer surgery on pain, physical, emotional and work- related functioning
}

\author{
An De Groef, ${ }^{1,2}$ Nele Devoogdt, ${ }^{1,2,3}$ Elien Van der Gucht, ${ }^{1}$ Lore Dams, ${ }^{4}$ \\ Koen Bernar, ${ }^{2,5}$ Lode Godderis, ${ }^{6,7}$ Bart Morlion,, Niamh Moloney, ${ }^{9,10}$ \\ Ann Smeets, ${ }^{11}$ Paul Van Wilgen, ${ }^{12,13,14}$ Mira Meeus ${ }^{4,12}$
}

To cite: De Groef A, Devoogdt N, Van der Gucht E, et al. EduCan trial: study protocol for a randomised controlled trial on the effectiveness of pain neuroscience education after breast cancer surgery on pain, physical, emotional and workrelated functioning. BMJ Open 2019;9:e025742. doi:10.1136/ bmjopen-2018-025742

- Prepublication history for this paper is available online. To view these files, please visit the journal online (http://dx.doi. org/10.1136/bmjopen-2018025742).

Received 31 July 2018 Revised 11 October 2018 Accepted 12 0ctober 2018

Check for updates

(c) Author(s) (or their employer(s)) 2019. Re-use permitted under CC BY-NC. No commercial re-use. See rights and permissions. Published by BMJ.

For numbered affiliations see end of article.

Correspondence to

Dr An De Groef;

an.degroef@kuleuven.be

\section{ABSTRACT}

Introduction Over the past decades, awareness on the importance of educational interventions in cancer pain management has increased. However, education is often restricted to biomedical pain management instructions. A more modern educational approach, also known as pain neuroscience education (PNE), explains pain from a biopsychosocial perspective. We hypothesise that this more comprehensive educational approach in the early treatment phase of breast cancer will lead to more beneficial effects for cancer pain management. Therefore, the aim of the present study is to investigate the effectiveness of this PNE intervention, in addition to best evidence physical therapy modalities for treatment and prevention of pain, physical, emotional and work-related functioning after breast cancer surgery, compared with a traditional biomedical educational intervention.

Methods A double-blinded randomised controlled trial has been started in November 2017 at the University Hospitals of Leuven. Immediately after breast cancer surgery, all participants $(n=184)$ receive a 12-week intensive standard physical therapy programme. They receive three additional refresher sessions at 6, 8 and 12 months postsurgery. In addition, participants receive three educational sessions during the first-month postsurgery and three 'booster sessions' at 6, 8 and 12 months postsurgery. In the intervention group, the content of the education sessions is based on the modern PNE approach. Whereas in the control group, the education is based on the traditional biomedical approach. The primary outcome parameter is pain-related disability 1 year after surgery. Secondary outcomes related to other dimensions of pain, physical, emotional and work-related functioning at 1-week, 4, 6, 8, 12 and 18 months postsurgery.

Ethics and dissemination The study will be conducted in accordance with the Declaration of Helsinki. This protocol has been approved by the ethical committee of the University Hospitals of Leuven. Results will be disseminated via peer-reviewed scientific journals and presentations at congresses.
Strengths and limitations of this study

- This study comprises a well-powered clinical trial investigating the additional effect of an easy deliverable pain neuroscience education intervention for pain-related disability and related outcomes following breast cancer treatment.

- A strength of the trial is the pragmatic nature of the study and applicability in daily clinical practice.

- The study is powered for the primary outcome parameter 'pain-related disability' 1 year after surgery.

Trial registration number NCT03351075.

\section{INTRODUCTION}

Breast cancer is the most frequent malignancy among women worldwide. ${ }^{1}$ Despite the high incidence, in Western countries, an increase in survival and life expectancy has been observed due to the ongoing improvement of detection method accuracy, early diagnosis and breast cancer treatment. ${ }^{1}$ Consequently, more attention is warranted towards the debilitating problems accompanying this disease and its treatment, which can persist for months or even years after diagnosis. In addition to fatigue, pain is the most frequent and persistent symptom following cancer and cancer treatment. Between $27 \%$ and $79 \%$ of women report pain 1 month after surgery, which is often attributed to local pain mechanisms caused by a postsurgery and/or radiotherapy tissue insult at that time point. ${ }^{2-5}$ One would expect the prevalence rate to diminish as healing occurs, yet this does not seem to be the case. In fact, $12 \%-82 \%$ of women 
still report persistent pain 1 year or later. ${ }^{4}$ This may indicate that besides local nociceptive and neuropathic pain mechanisms, a third pain mechanism characterised by altered nociceptive processing without clear evidence of persistent tissue damage causing the activation of peripheral nociceptors (ie, nociceptive pain) or evidence for disease or lesion of the somatosensory system causing the pain (ie, neuropathic pain) ${ }^{6-8}$ Moreover, pain interferes with pain, physical, emotional and work-related disability and therefore severely prejudices a person's quality of life and participation in society. ${ }^{9-11}$ Hence, adequate pain management in the early stage of breast cancer treatment is necessary to prevent and improve pain and pain-related disability, both at short and long term.

Despite the effectiveness of currently applied physical therapy modalities after breast cancer surgery (such as manual techniques, specific exercises and general exercises), up to $72 \%$ of women still experience pain and the resulting disabilities after finishing breast cancer treatment. ${ }^{12}$ Over the past decades, awareness on the important role of educational interventions in the management of cancer pain has increased. ${ }^{13-15}$ These general educational interventions have been shown to be effective for improving pain severity, self-efficacy and knowledge and attitude to pain and analgesia in patients with cancer. However, effect sizes are only moderate and of limited clinical relevance. ${ }^{13}$ This can be explained by the fact that these educational interventions mainly focus on tissue and tissue injury as the source of pain and are often restricted to biomedical pain management instructions and general advice on physical activity and analgesics. ${ }^{13-15}$ They focus on explaining treatment side effects and improving patients' coping strategies. Recently, increased knowledge on pain mechanisms has led to a more modern educational approach, also known as pain neuroscience education (PNE) ${ }^{16-19}$ This explains the neurophysiology of chronic pain and the ability of the nervous system to modulate pain experience, as well as the potential influences of sleep, thoughts, feelings and culture, among others, on pain. Thereby, it targets a reconceptualisation from a biomedical or structural model to an actual biopsychosocial model of pain. Through the knowledge that pain is often an unreliable indicator of the presence or extent of tissue damage and if patients may become open to exploring broader contributions to pain, pain-related behaviour might change by shifting from passive therapy receiving to active self-management. Increased knowledge of the broad contributions to pain, ${ }^{4}$ as well as awareness of different pain mechanisms following breast cancer treatment ${ }^{6-8}$ provides justification for the integration of PNE in this population. Applying PNE could enhance the effectiveness of the currently applied physical therapy modalities for prevention and treatment of pain and related disabilities after breast cancer treatment, compared with a traditional biomedical educational intervention. Indeed, encouraging people to address emotional, cognitive and broader health-related factors in the early stage of cancer treatment may enhance recovery during and after the treatment. To our knowledge, only one controlled trial investigated the effectiveness of PNE in the early stage of breast cancer treatment. ${ }^{20}$ Although the results were very promising for shoulder function, only short-term effects were examined, no randomisation was performed and no pain-related or other health-related outcomes were evaluated.

\section{Objectives}

The main scientific objective is to examine the effectiveness of PNE, in addition to a standard best evidence physical therapy programme, on pain, physical, emotional and work-related functioning in the early stage of breast cancer treatment, compared with a traditional biomedical educational intervention, up to 1.5 years after surgery (EduCan Trial). This will be performed through a doubleblinded randomised controlled trial.

\section{METHODS AND ANALYSIS}

Described according to the Standard Protocol Items: Recommendations for Interventional Trials guidelines (http://www.spirit-statement.org/protocol-version/).

\section{Trial design and study setting}

A parallel, two-arm randomised controlled trial with blinding of assessors and physical therapists providing the standard physical therapy programme in both arms and masking of the participants. The trial started in November 2017 at the Department of Physical Medicine and Rehabilitation of the University Hospitals in Leuven (Belgium). A schedule of the EduCan Trial is provided in table 1.

\section{Patient and public involvement in the trial design}

One female patient with breast cancer and a representative of the National Health Service were consulted during the initial grant preparation and trial set-up. The patient representative provided valuable insight into the worries and concerns experienced during cancer treatment. The representative of the National Health service contributed to the design of the study and advised on assessment of work-related functioning outcomes.

\section{Eligibility criteria}

Women are eligible to participate in the EduCan Trial if they are scheduled for surgery for breast cancer at the Multidisciplinary Breast Centre of the University Hospitals of Leuven. Patients with increased risk of developing pain after breast cancer surgery are included..$^{21-23}$ Therefore, inclusion criteria are: (1) diagnosed with histologically confirmed invasive or non-invasive primary breast cancer, (2) scheduled for surgical excision including either axillary lymph node dissection and mastectomy (whether or not in combination with reconstructive surgery) or breast conserving; or either sentinel node biopsy and mastectomy (whether or not in combination with reconstructive surgery); (3) aged 18 years or older; 
Table 1 Schedule of enrolment, interventions and assessments of the EduCan Trial

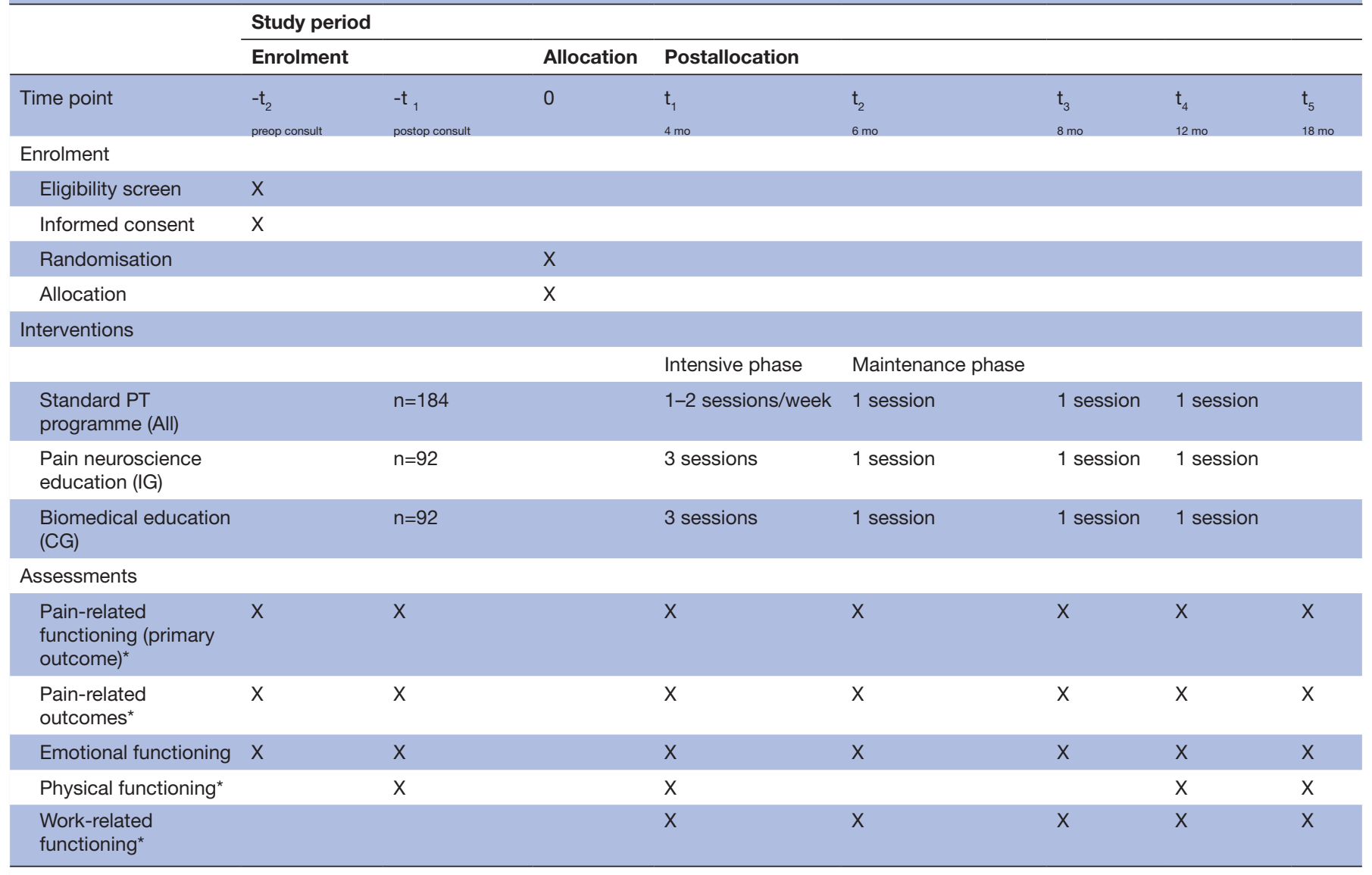

*See table 2 for details on the content of the different assessments at each point in time.

CG, control group; IG, intervention group; Mo, months.

(4) can comply with the study protocol. Patients with active metastasis are excluded because of the higher risk of mortality.

\section{Participant screening, recruitment and consent}

Participants are identified from scheduled operation lists and screened for eligibility criteria. The initial screening process is undertaken by a member of the research team. Potentially eligible participants are approached and recruited during the preoperative consult at the Multidisciplinary Breast Centre of the University Hospitals of Leuven. All eligible patients receive an information sheet and the explanation of the study during the preoperative consult. Next, they are asked to have a preoperative baseline measurement for which a separate informed consent exists. Because of ethical and deontological reason, patients will not be forced to decide on participation in the complete EduCan Trial at this moment, but initially only for the baseline measurements.

During their postoperative hospital stay, a member of the research team will meet the eligible participants again, answer further questions and include them in the further trial if wanted. Then, a second informed consent is signed for participating in the complete EduCan Trial. The preoperative baseline measurement of non-participating patients will be stored in the medical file of the patient and can be consulted on clinical follow-up appointments to evaluate the recovery of the patient but is not used for research purpose. The participants' flow is summarised in figure 1.

\section{Allocation and randomisation}

Therapists and assessors are blinded to the allocation of the treatment groups. The therapists providing the standard physical therapy programme will be unaware of the type of education received by the patient (PNE in the intervention group and biomedically focused education in the control group). Consequently, they give therapy in both groups. Assessors are blinded to the maximal extent possible. With regard to this, patients are asked not to communicate with the assessors about the intervention received. Patients are masked for the allocation to the intervention/control group; they do not know which one is the experimental intervention and which one is the control intervention, however, they will, of course, be aware of the intervention received. To reduce bias, within one participant, therapists giving the standard physical therapy programme, therapists given the educational intervention and the assessors are all different persons.

At the end of the trial, the success of assessor blinding will be examined by asking whether the assessor thought the participant had received the experimental or control 
Screening and Recruitment

Eligible breast cancer patients are identified from operation lists at the Multidisciplinary Breast Center of the University Hospitals Leuven (Belgium).

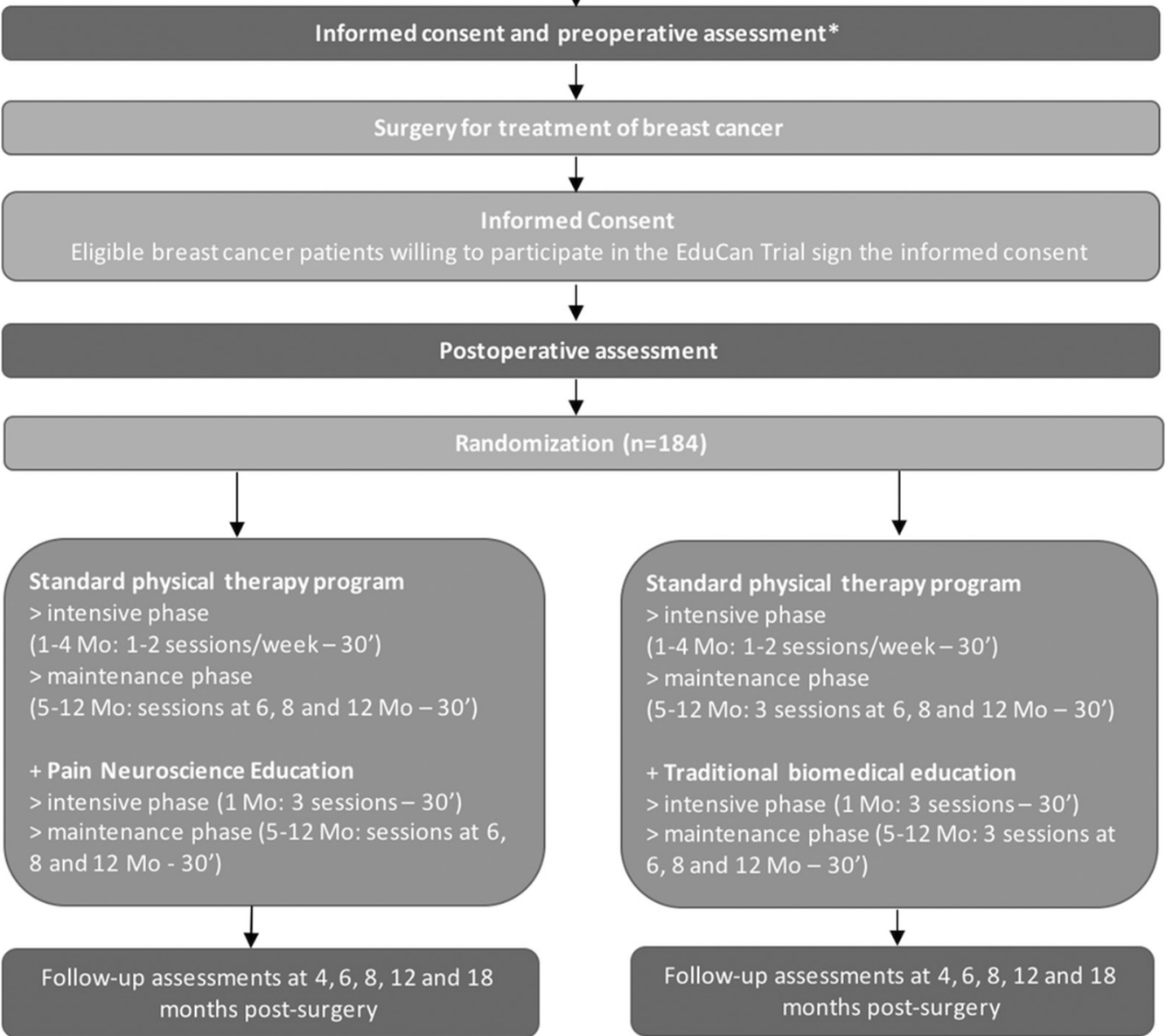

Figure 1 Flow diagram of the EduCan Trial. *A separate informed consent is available for the preoperative assessment. Mo, months.

intervention, including the percentage of certainty (ie, $50 \%$ certainty means a pure guess). The same will be done for patient masking. The research members performing statistical analysis will be blinded as well.

The randomisation is computer generated and is performed by using permuted blocks (size $=4$ ). An independent coworker at the department carries out the randomisation to ensure blinding of the research team. The sequence of randomisation is determined by the patient's identification number, which she receives after signing informed consent. Participants are randomised in a 1:1 ratio between intervention and control arms.

\section{Interventions}

Standard physical therapy programme

All participants in the EduCan Trial attend a standard physical therapy programme. The standard physical therapy programme is based on currently available evidence and clinical experience of the research team and will include three physical therapy modalities. Additionally, to avoid conflicts with the information given during the educational interventions, a communication sheet had been made. This document contains guidelines on which information the physical therapists can provide on common topics discussed during the standard physical therapy sessions. First, manual techniques including (1) passive mobilisations to restore shoulder range of motion, (2) stretching of the pectoral muscles to improve muscle flexibility and (3) scar tissue massage to improve flexibility of the scar(s) will be implemented. $^{122425}$ Second, specific exercises to improve shoulder range of motion and upper limb strength have been proven to be effective for the treatment of upper limb problems after breast cancer and will start immediately after surgery as well. ${ }^{12} 26$ Specific exercises are instructed during the individual session and continued at home. Third, patients are advised on general exercises. General exercises should be implemented to increase the patient's physical activity level. In general, these recommendations consist of physical activity at a minimum level of moderate intensity over an extended 
period and can include, for example, running, walking, cycling, swimming. ${ }^{27} 28$

During month 1-4, an intensive physical therapy programme is implemented because of the postoperative side effects. Patients will attend 1-2 individual sessions of $30 \mathrm{~min}$ per week during the intensive phase, starting 1-week postsurgery. All patients start with a frequency of two sessions per week, decreasing to once every 2 weeks. The decrease in the frequency of the sessions is pragmatically chosen based on the individual progression and need of the patient.

Up to 1 year after surgery, a maintenance physical therapy programme is implemented to follow up on the exercises performed at home and to treat possible additional/new side effects of other adjuvant treatment modalities such as radiotherapy, chemotherapy and hormone therapy. An individual maintenance session of $30 \mathrm{~min}$ is scheduled 6,8 and 12 months postsurgery.

Additionally, information about prevention of lymphoedema is given by a specialised physical therapist: about normal use of the upper limb, avoiding pinching off the arm, skin care and control of body weight. ${ }^{29}$ One group information sessions of $60 \mathrm{~min}$ on this topic is organised each month which should be attended once by every participant (both patients from the intervention and control group together) and this as soon as possible after surgery. Patients also receive a brochure with this information. If patients develop lymphoedema, they are additionally referred to the Lymphovenous Center of the University Hospitals of Leuven for further treatment of the lymphoedema.

\section{Educational intervention}

The educational sessions are individual and last for $30 \mathrm{~min}$. The first PNE session is given within the first postoperative week before the start of the standard physical therapy programme to prepare the patient for the physical therapy sessions. Information is presented verbally (explanation by the therapist) and in multimedia forms (power point presentation with summaries, pictures, metaphors and diagrams on a computer). After the first session, patients also receive an information leaflet on paper and are asked to read it carefully at home. They also receive a web-link to an online presentation that summarises the provided information. Additional written information that can be read afterwards is a valuable and essential part of the educational intervention. In the following 4 weeks after surgery, two additional PNE sessions are provided to ensure that the patient understands the pain physiology and principles of activity management and can relate this to the physical therapy programme and his/her pain complaint. However, education is a continuous process initiated at the start and continuing into and followed up during the longer term rehabilitation programme. Therefore, three additional booster sessions are organised at 6 , 8 and 12 months postsurgery. During the booster sessions, the information given postoperatively will be rehearsed and application of the information into future stages of the recovery process will be discussed. Special attention is given to return to preoperative activities and return to work (if applicable). Regarding this, a second information leaflet on paper will be given to the patient. Patients in the control arm and intervention arm will have the same schedule of educational sessions, only the content of the education differs from the intervention arm.

\section{Intervention arm: PNE}

Based on the available literature, a modern PNE programme has been established to explain pain specifically for this population. ${ }^{131520}$ The content and pictures of the educational sessions are based on the book 'Explain Pain', 30 'Pijneducatie een praktische handleiding voor (para)medici' (Van Wilgen and Nijs, 2011) and the 'The Pain Toolkit', ${ }^{31}$ as used in earlier studies. ${ }^{32} 33$ Topics addressed during the PNE sessions will include: the characteristics of acute versus chronic pain; specific side effects of the different breast cancer treatment modalities in relation to pain; how pain is a product of the brain; how pain becomes chronic (plasticity of the nervous system, modulation, modification, central sensitisation); potential sustaining factors of pain such as emotions, stress, pain cognitions and pain behaviour.

Additionally, this PNE intervention includes advice for activity management, while experiencing pain and other symptoms. In addition to the general recommendations for general exercise and advice to stay active in the standard physical therapy programme, the PNE guides patients in performing general exercises and activities according to the graded activity principle. Graded activity is applied according to the guidelines reported by the International Association for the Study of Pain. ${ }^{34}$ This includes general exercise activities according to pacing strategies for 'persisters' (ie, restructuring the activity pattern to avoid peaks of overactivity and exacerbations of their pain) and graded activity for 'avoiders' (ie, time-contingent increase of physical activity). PNE is crucial here to help patients interpret pain during exercise in the correct context. Finally, advice on returning to work in the context of present pain complaints and how to apply the principles described above for activity management can be applied in the working situation will be provided.

\section{Control arm: traditional biomedical education}

Traditional biomedical educational interventions consist of explaining patient's pain experience in relation to the therapeutic procedures from a tissue and biomechanical perspective. ${ }^{35}{ }^{36}$ Information on the different side effects of surgery, radiotherapy, chemotherapy, hormone therapy and target therapy is given. The role of different structures and injured versus healthy tissue in acute and persistent pain is discussed. Pain is explained from a biomechanical point of view, for example, deviance from normal expected movement patterns and postures. Additionally, during the educational sessions and rehabilitation program, patients receive advice on activity 
management. This advice is to stay active as minimally possible during treatment and increase their physical activity level according to current recommendations for general exercises after treatment. Based on the American Cancer Society Guidelines on Physical Activity at least $150 \mathrm{~min}$ of moderate intensity (heart rate $50 \%-70 \%$ of the maximum heart rate or a score of 12-14 on Borg Rating of Perceived Exertion (RPE)) or 75 min of vigorous intensity activity $(70 \%-85 \%$ of the maximum heart rate or RPE of $>15$ ) each week (or a combination of these), preferably spread throughout the week is recommended. Finally, advice on returning to work in the context of the different (persistent) side effects of the treatments will be provided.

\section{Outcomes}

The outcome measures were chosen in accordance with the guideline for core outcome domains to be used in clinical trials on multimodal treatment approaches for pain as advocated by an international steering committee (VAPAIN (Validation and Application of a patient-relevant core set of outcome domains to assess multimodal PAIN therapy) recommendations) ${ }^{37}$ and the
IMMPACT (Initiative on Methods, Measurement, and Pain Assessment in Clinical Trials) recommendations for the outcome measures in pain clinical trials. ${ }^{38}$

The primary outcome is pain-related functioning at 12 months measured using the Pain Disability Index (PDI). Secondary outcomes are other pain symptoms and characteristics, physical functioning, emotional functioning and work-related functioning. Additionally, a number of visits are recorded. Assessments are performed within 1-week preoperatively, within 1-week postoperatively and then at 4, 6, 8, 12 and 18 months after surgery. However, because of feasibility limitations, not all outcome parameters are assessed at each assessment time point. Tables 1 and 2 present the study outcome measures by assessment time point. In table 3 , the outcome measures are described in more detail.

\section{Sample size}

A power calculation was performed by the Leuven Biostatistics and statistical bioinformatics Centre of KU Leuven (Katholieke Universiteit Leuven) for the primary outcome parameter 'PDI after 1 year'. Sample size calculation was based on data available in the literature for the

Table 2 Study outcome measures by assessment time point

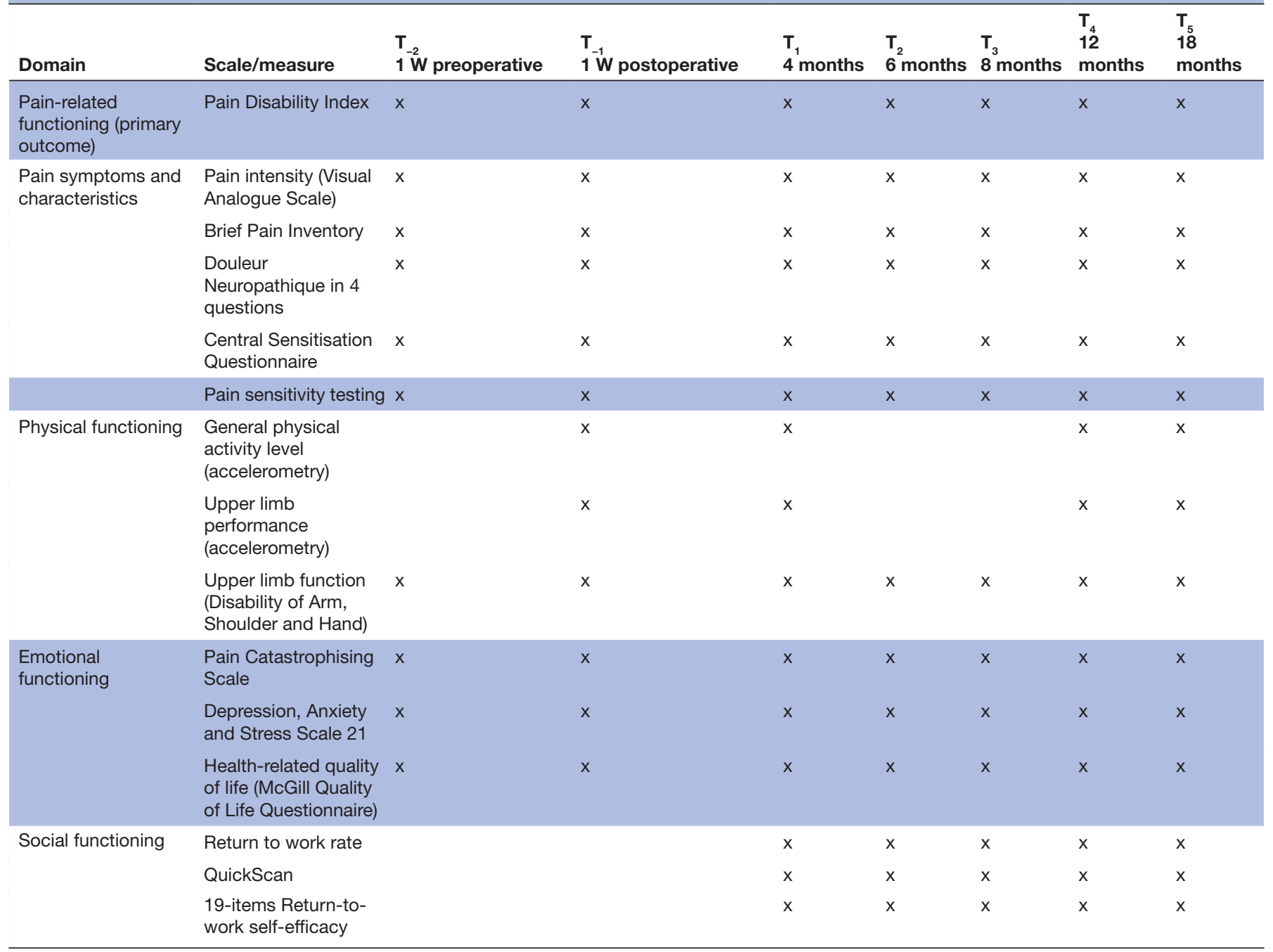


Table 3 Outcome measures of the EduCan Trial

\begin{tabular}{ll}
\hline Outcome & Assessment method \\
\hline Pain-related functioning (primary outcome) & $\begin{array}{l}\text { Pain Disability Index (PDI). The PDI is a short, self-reported questionnaire for measuring the degree of interference of } \\
\text { pain with normal role functioning (family/home responsibilities, recreation, social activity, occupation, sexual behaviour, } \\
\text { self-care, and life-support activity). }{ }^{39} 40\end{array}$
\end{tabular}

Pain symptoms and characteristics

Pain intensity Visual Analogue Scale. Present pain intensity and mean pain intensity during the past week for pain at the upper limb region (ie, shoulder-neck region, arm, axilla, trunk side and breast region).
Brief pain inventory Medication use, pain quality, pain location, pain severity and response to treatment. ${ }^{41}$
Presence of neuropathic pain Douleur Neuropathique in 4 questions (DN4). The DN4 a questionnaire generating reliable and valid data for identifying pain of predominantly neuropathic origin. ${ }^{42}$

Presence of hypersensitivity of Central Sensitisation Inventory (CSI). The CSI is a questionnaire generating reliable and valid data to assess symptoms the central nervous system related to central sensitisation mechanisms. ${ }^{43}$

Pain sensitivity testing

Quantitative sensory testing: mechanical detection and pain thresholds*

Quantitative sensory testing: temperature detection and pain thresholds*

Quantitative sensory testing: pressure pain thresholds*

Twelve monofilaments (MARSTOCK nerve test-Optihair2, Schriesheim, Germany) with a force from 0.25 to $512 \mathrm{mN}$ are used. The mechanical detection threshold is defined as the lowest mechanical force that the participant can detect. The mechanical pain threshold is defined as the lowest mechanical force that the participant perceives as painful or unpleasant. Monofilaments are applied with a rate of $2 \mathrm{~s}$ 'on' and $2 \mathrm{~s}$ 'off' at the inner side of the upper arm and lateral trunk side.

The computerised thermotest device TSA-II-NeuroSensory Analyser is used. The method of limits is used. The detection and pain thresholds are measured as the first identified stimulus under increasing stimulus intensities. The participant has to push the button once the stimulus is detected or perceived as painful or unpleasant. This is repeated three times for each threshold. The mean of three stimuli for each threshold is calculated and used for analysis. ${ }^{44}$

Measured by a digital Wagner FPX algometer. Points of measurement are defined by palpation for most tender muscle points (one per muscle) at the major pectoral muscle region, the lateral trunk side and upper trapezius muscle region. The participant is asked to say 'stop' when the sensation of pressure first changes to pain. The mean value of the two measurements is calculated and used for analysis. ${ }^{45}$

Presence of widespread pain/secondary hyperalgesia

Presence and degree of impaired nociceptive inhibitory mechanisms (ie, conditioned pain modulation (CPM))

Quantitative sensory testing is performed both at the local painful area as at remote body parts (ie, quadriceps muscle at the non-affected side) and pain distribution is displayed on a body diagram.

Assessment of conditioned pain modulation will be done using the Medoc two thermode Q-Sense CPM system. This system involves a 'test' stimulus and a 'conditioning' stimulus applied on the ulnar side of the forearms. The test stimulus (at the affected side) is used to assess pain sensitivity to a warmth stimulus pre and post the noxious conditioning stimulus and the difference is calculated between premeasures and postmeasures. When the second pressure pain threshold (ie, test stimulus) is similar or lower than the first, dysfunctional inhibitory pain mechanisms are present. ${ }^{46} 47$

Presence and degree of enhanced facilitation mechanisms (ie, wind-up)

Wind-up of pain or temporal summation will be assessed by applying repetitive nociceptive stimulation with a $26 \mathrm{~g}$ nylon monofilament at the major pectoral muscle at the affected side. The perceived intensity of the stimulus (the first, the last and aftersensations) are reported by using a Numeric Rating Scale. The temporal summation value is calculated as the difference between the first and the last stimuli or the slope of the increase in pain intensity. A response for enhanced temporal summation is deemed positive if participants perceive the initial stimulus as non-noxious, but it becomes noxious, increasing by at least two points on a Numeric Rating Scale, or if baseline pain intensity increases by at least two points. ${ }^{46-48}$

Presence and degree of hypersensitivity to nonmechanical stimuli

The Central Sensitisation Inventory, a questionnaire generating reliable and valid data to assess symptoms related to central sensitisation mechanisms. ${ }^{4-51}$

Physical functioning

General physical activity and upper limb performance

Three ActiLife accelerometers, one on the pelvis (seven consecutive days) and one on each wrist (three consecutive days), will be worn during waking hours. Outcome parameters are general activity level, unimanual/bimanual time and intensity of both unimanual/bimanual use. The ActiLife V.6.9.5 Firmware V.2.2.1 will be used to save raw data. Data will be further processed with Matlab, using custom-written routines. ${ }^{52} 53$

Upper limb function DASH questionnaire. The DASH is a self-reported questionnaire on upper limb function. ${ }^{3}$

Emotional functioning

Pain catastrophising

Depression, anxiety and stress

Pain Catastrophising Scale (PCS). The PCS is a self-reported questionnaire measuring catastrophic thinking related to pain. ${ }^{54}$

Depression Anxiety Stress scales 21 (DASS-21). The DASS-21 is a self-reported questionnaire that measures the three related states of depression, anxiety and stress. ${ }^{55}$

Health-related quality of life McGill Quality of Life questionnaire. ${ }^{56}$

Social functioning

Return to work rate

Self-reported questionnaire on return to work, employment status, work adjustments. 
Table 3 Continued

\begin{tabular}{ll}
\hline Outcome & Assessment method \\
\hline QuickScan & $\begin{array}{l}\text { Questionnaire on health status and return-to-work obstacles in order to assess potential predictive factors for long-term } \\
\text { absenteeism. }\end{array}$ \\
$\begin{array}{l}\text { Patients perceived ability to } \\
\text { work }\end{array}$ & $\begin{array}{l}\text { 19-item Return-to-work self-efficacy questionnaire (RTWSE-19). The RTWSE-19 is a self-reported questionnaire on the } \\
\text { patients' perceived ability to work. }\end{array}$
\end{tabular}

${ }^{*}$ Testing is performed bilaterally, except preoperatively because of feasibility reasons. CPM, Conditioned Pain Modulation.

PDI. ${ }^{39} 40$ and calculated to detect with $80 \%$ power a difference of $20 \%$ in PDI after 1 year. Assuming a coefficient of variation $(\mathrm{CV})$ equal to $0.5,87$ participants per group are needed based on a two-sample pooled t-test of a mean ratio with lognormal data and setting alpha equal to 0.05 . The assumed CV is a conservative estimate, derived from the observed CV of 0.30 in a sample of normative data for women with chronic pain. To anticipate a drop-out rate of approximately 5\%, 184 participants in total will be recruited. The drop-out rate is based on previous similar trials at our institution. ${ }^{2425} 29$ To handle the potential missing measurements after 1 year, the comparison of the PDI will be based on a multivariate normal model for longitudinal measurements fitted on all repeated measures over time (preoperative, postoperative, 4, 6, 8, 12 and 18 months). A log-transformation will be applied if necessary to handle the right-skewed distribution of the PDI.

\section{Data analysis}

Statistical analysis will be intention to treat and will comply with the Consolidated Standards of Reporting Trials (CONSORT) guidelines. Analysis will be conducted in a blinded way. The continuous data will be summarised using mean, SD, median and range values. The primary outcome will be analysed using multilevel linear regression models for repeated (longitudinal) measures, using an unstructured covariance matrix. The mean change from baseline (ie, preoperative assessment) to 4, 6, 8, 12 and 18 months (with correction for the postoperative assessment) will be estimated using contrast statements for each of the treatment arms. The difference in mean changes and their $95 \%$ CIs between interventions will be plotted graphically so that change can be assessed over the course of the study. Continuous secondary outcomes will be assessed in a similar way to the primary outcome. Categorical data will be analysed using logistic models. For non-repeated continuous and binary measurements, ordinary linear regression and logistic models will be used, respectively.

\section{Data security and management}

Participant data are stored on a secure database in accordance with the General Data Protection Regulations (2018). Data are de-identified and a unique trial identification number used on all participant communication. Clinical and patient forms are being checked for completeness and congruity before data entry onto the database. Data will undergo additional checks to ensure consistency between data submitted and original paper forms. Trial documentation and data will be archived for at least 10 years after completion of the trial.

\section{Trial monitoring}

The steering committee of the research team will oversee all aspects of design, delivery, quality assurance and data analysis. The steering committee will monitor the trial at least once per year.

\section{ETHICS AND DISSEMINATION}

The EduCan Trial applies the principles established in the Declaration of Helsinki. Participants provide written informed consent before data collection. Only de-identified coded and interpreted data will be shared between the members of the research team.

\section{Dissemination of results}

The research team are committed to full disclosure of the results of the trial. Findings will be reported in accordance with CONSORT guidelines and we aim to publish in high impact journals. Given the multitude of outcome parameters, results will be divided over several papers. Our patient representatives and representative of the National Health Service will assist with dissemination of study results. The funder will take no role in the analysis or interpretation of trial results.

\section{Author affiliations}

${ }^{1}$ Department of Rehabilitation Sciences, KU Leuven - University of Leuven, Leuven, Belgium

${ }^{2}$ Department of Physical Medicine and Rehabilitation, University Hospitals Leuven, Leuven, Belgium

${ }^{3}$ Department of Vascular Surgery, University Hospitals Leuven, Leuven, Belgium ${ }^{4}$ Department of Rehabilitation Sciences and Physiotherapy, Faculty of Medicine and Health Sciences, University of Antwerp, Antwerp, Belgium

${ }^{5}$ The Leuven Centre for Algology and Pain Management, University Hospitals Leuven, Leuven, Belgium

${ }^{6}$ Centre for Environment and Health of KU Leuven, Leuven, Belgium

${ }^{7}$ IDEWE, External Service for Prevention and Protection at Work, Leuven, Belgium

${ }^{8}$ Department of Cardiovascular Sciences, Section Anaesthesiology and Algology, KU Leuven - University of Leuven, Leuven, Belgium

${ }^{9}$ Faculty of Medicine and Health Sciences, Macquarie University, Sydney, New South Wales, Australia

${ }^{10}$ Thrive Physiotherapy, Guernsey, UK

${ }^{11}$ Department of Surgical Oncology, University Hospitals Leuven, Leuven, Belgium

${ }^{12}$ Pain in Motion research group (www.paininmotion.be)

${ }^{13}$ Department of Physiotherapy, Human Physiology and Anatomy, Faculty of Physical Education and Physiotherapy, Vrije Universiteit Brussel, Brussels, Belgium 
${ }^{14}$ Transcare, Transdisciplinary Pain Management Centre, Groningen, The Netherlands

Acknowledgements The authors would like to thank all the trial participants. The authors would also like to thank all the physical therapy staff, surgical oncology teams, breast cancer nurses and research departments collaborating on this study.

Contributors ADG, EVdG and LD drafted the manuscript. ADG, ND, MM, AS, LG, BM, KB, NM and PVW are the principal coordinators of the EduCan Trial. All other authors contributed to the establishment of the protocol, revised the manuscript and provided input according to their area of expertise.

Funding The study is funded by Research Foundations - Flanders (FWO) (T005117N).

\section{Competing interests None declared.}

Patient consent for publication Obtained.

Ethics approval Ethics approval was granted by the local Ethical Committee of the University Hospitals Leuven (s60702).

Provenance and peer review Not commissioned; externally peer reviewed.

Open access This is an open access article distributed in accordance with the Creative Commons Attribution Non Commercial (CC BY-NC 4.0) license, which permits others to distribute, remix, adapt, build upon this work non-commercially, and license their derivative works on different terms, provided the original work is properly cited, appropriate credit is given, any changes made indicated, and the use is non-commercial. See: http://creativecommons.org/licenses/by-nc/4.0/.

\section{REFERENCES}

1. Ferlay J, Soerjomataram I, Dikshit R, et al. Cancer incidence and mortality worldwide: sources, methods and major patterns in GLOBOCAN 2012. Int J Cancer 2015;136:E359-86.

2. Rietman JS, Dijkstra PU, Geertzen JH, et al. Short-term morbidity of the upper limb after sentinel lymph node biopsy or axillary lymph node dissection for Stage I or II breast carcinoma. Cancer 2003;98:690-6.

3. Hidding JT, Beurskens CH, van der Wees PJ, et al. Treatment related impairments in arm and shoulder in patients with breast cancer: a systematic review. PLoS One 2014;9:e96748.

4. Wang L, Guyatt GH, Kennedy SA, et al. Predictors of persistent pain after breast cancer surgery: a systematic review and meta-analysis of observational studies. CMAJ 2016;188:E352-61.

5. Leysen L, Beckwée D, Nijs J, et al. Risk factors of pain in breast cancer survivors: a systematic review and meta-analysis. Support Care Cancer 2017;25:3607-43.

6. De Groef A, Meeus M, De Vrieze T, et al. Unraveling self-reported signs of central sensitization in breast cancer survivors with upper limb pain: prevalence rate and contributing factors. Pain Physician 2018;21:E247-56.

7. Nijs J, Leysen L, Adriaenssens N, et al. Pain following cancer treatment: Guidelines for the clinical classification of predominant neuropathic, nociceptive and central sensitization pain. Acta Oncol 2016;55:659-63.

8. Nijs J, Leysen L, Pas R, et al. Treatment of pain following cancer: applying neuro-immunology in rehabilitation practice. Disabil Rehabil 2018;40:714-21.

9. Kudel I, Edwards RR, Kozachik S, et al. Predictors and consequences of multiple persistent postmastectomy pains. J Pain Symptom Manage 2007;34:619-27.

10. Sun Y, Shigaki CL, Armer JM. Return to work among breast cancer survivors: a literature review. Support Care Cancer 2017;25:709-18.

11. Shin WK, Song S, Jung SY, et al. The association between physical activity and health-related quality of life among breast cancer survivors. Health Qual Life Outcomes 2017;15:132.

12. De Groef A, Van Kampen M, Dieltjens E, et al. Effectiveness of postoperative physical therapy for upper-limb impairments after breast cancer treatment: a systematic review. Arch Phys Med Rehabil 2015;96:1140-53.

13. Bennett MI, Bagnall AM, José Closs S. How effective are patientbased educational interventions in the management of cancer pain? Systematic review and meta-analysis. Pain 2009;143:192-9.

14. Armes J, Chalder T, Addington-Hall J, et al. A randomized controlled trial to evaluate the effectiveness of a brief, behaviorally oriented intervention for cancer-related fatigue. Cancer 2007;110:1385-95.

15. Ling CC, Lui LY, So WK. Do educational interventions improve cancer patients' quality of life and reduce pain intensity? Quantitative systematic review. J Adv Nurs 2012;68:511-20.
16. Moseley GL, Butler DS. Fifteen years of explaining pain: the past, present, and future. J Pain 2015;16:807-13.

17. Nijs J, Meeus M, Van Oosterwijck J, et al. Treatment of central sensitization in patients with 'unexplained' chronic pain: what options do we have? Expert Opin Pharmacother 2011;12:1087-98.

18. Nijs J, Paul van Wilgen C, Van Oosterwijck J, et al. How to explain central sensitization to patients with 'unexplained' chronic musculoskeletal pain: practice guidelines. Man Ther 2011;16:413-8.

19. Nijs J, Torres-Cueco R, van Wilgen CP, et al. Applying modern pain neuroscience in clinical practice: criteria for the classification of central sensitization pain. Pain Physician 2014;17:447-57.

20. Sato $F$, Ishida $T$, Ohuchi $N$. The perioperative educational program for improving upper arm dysfunction in patients with breast cancer: a controlled trial. Tohoku J Exp Med 2014;232:115-22.

21. Andersen KG, Kehlet H. Persistent pain after breast cancer treatment: a critical review of risk factors and strategies for prevention. J Pain 2011;12:725-46.

22. De Groef A, Van Kampen M, Tieto E, et al. Arm lymphoedema and upper limb impairments in sentinel node-negative breast cancer patients: A one year follow-up study. Breast 2016;29:102-8.

23. Katz J, Poleshuck EL, Andrus $\mathrm{CH}$, et al. Risk factors for acute pain and its persistence following breast cancer surgery. Pain 2005;119(13):16-25.

24. De Groef A, Van Kampen M, Vervloesem N, et al. Effect of myofascial techniques for treatment of persistent arm pain after breast cancer treatment: randomized controlled trial. Clin Rehabil 2018;32:451-61.

25. De Groef A, Van Kampen M, Vervloesem N, et al. Myofascial techniques have no additional beneficial effects to a standard physical therapy programme for upper limb pain after breast cancer surgery: a randomized controlled trial. Clin Rehabil 2017;31:1625-35.

26. McNeely ML, Campbell K, Ospina M, et al. Exercise interventions for upper-limb dysfunction due to breast cancer treatment. Cochrane Database Syst Rev 2010;6:CD005211.

27. Zeng Y, Huang M, Cheng AS, et al. Meta-analysis of the effects of exercise intervention on quality of life in breast cancer survivors. Breast Cancer 2014;21:262-74.

28. Mishra SI, Scherer RW, Geigle PM, et al. Exercise interventions on health-related quality of life for cancer survivors. Cochrane Database Syst Rev 2012;8:Cd007566.

29. Devoogdt N, Christiaens MR, Geraerts I, et al. Effect of manual lymph drainage in addition to guidelines and exercise therapy on arm lymphoedema related to breast cancer: randomised controlled trial. BMJ 2011;343:d5326.

30. Thacker M. Explain Pain. By David Butler and G. Lorimor Moseley. Noigroup, Adelaide, 2003, 129 pp. ISBN 097509100 X: Whurr Publishers Ltd, 2004:185-7.

31. Moore P, Cole F. Pain Toolkit. 2002 www.paintoolkit.org.

32. Moseley L. Combined physiotherapy and education is efficacious for chronic low back pain. Aust J Physiother 2002;48:297-302.

33. Van Oosterwijck J, Meeus M, Paul L, et al. Pain physiology education improves health status and endogenous pain inhibition in fibromyalgia: a double-blind randomized controlled trial. Clin J Pain 2013;29:873-82.

34. Mira Meeus JN, Wilgen P, Noten S, et al. Moving on to movement in patients with chronic joint pain. Pain Clinical Updates 2016.

35. Louw A, Puentedura EJ, Zimney K, et al. Know pain, know gain? A perspective on pain neuroscience education in physical therapy. $J$ Orthop Sports Phys Ther 2016;46:131-4.

36. Louw A, Zimney K, Puentedura EJ, et al. The efficacy of pain neuroscience education on musculoskeletal pain: a systematic review of the literature. Physiother Theory Pract 2016;32:332-55.

37. Kaiser U, Kopkow C, Deckert S, et al. Developing a core outcomedomain set to assessing effectiveness of interdisciplinary multimodal pain therapy: the VAPAIN consensus statement on core outcomedomains. Pain 2017

38. Dworkin RH, Turk DC, Farrar JT, et al. Core outcome measures for chronic pain clinical trials: IMMPACT recommendations. Pain 2005;113:9-19.

39. Soer R, Reneman MF, Vroomen PC, et al. Responsiveness and minimal clinically important change of the Pain Disability Index in patients with chronic back pain. Spine 2012;37:711-5.

40. Chibnall JT, Tait RC. The Pain Disability Index: factor structure and normative data. Arch Phys Med Rehabil 1994;75:1082-6.

41. Cleeland CS, Ryan KM. Pain assessment: global use of the Brief Pain Inventory. Ann Acad Med Singapore 1994;23:129-38.

42. Bouhassira D, Attal N, Alchaar $\mathrm{H}$, et al. Comparison of pain syndromes associated with nervous or somatic lesions and development of a new neuropathic pain diagnostic questionnaire (DN4). Pain 2005;114:29-36. 
43. Kregel J, Vuijk PJ, Descheemaeker F, et al. The Dutch Central Sensitization Inventory (CSI): factor analysis, discriminative power, and test-retest reliability. Clin J Pain 2016;32:624-30.

44. Mucke M, Cuhls H, Radbruch L, et al. Quantitative sensory testing (QST). Schmerz 2016.

45. De Groef A, Van Kampen M, Vervloesem N, et al. Inter-rater reliability of shoulder measurements in middle-aged women. Physiotherapy 2017; 103:222-30.

46. Meeus M, Ickmans K, Struyf F, et al. Does acetaminophen activate endogenous pain inhibition in chronic fatigue syndrome/fibromyalgia and rheumatoid arthritis? A double-blind randomized controlled cross-over trial. Pain Physician 2013;16:E61-70.

47. Nahman-Averbuch $\mathrm{H}$, Yarnitsky D, Granovsky $\mathrm{Y}$, et al. Pronociceptive pain modulation in patients with painful chemotherapy-induced polyneuropathy. J Pain Symptom Manage 2011;42:229-38.

48. Edwards RR, Mensing G, Cahalan C, et al. Alteration in pain modulation in women with persistent pain after lumpectomy: influence of catastrophizing. J Pain Symptom Manage 2013;46:30-42.

49. Mayer TG, Neblett R, Cohen H, et al. The development and psychometric validation of the central sensitization inventory. Pain Pract 2012;12:276-85.
50. Scerbo T, Colasurdo J, Dunn S, et al. Measurement properties of the central sensitization inventory: a systematic review. Pain Pract 2018;18:544-54.

51. Cuesta-Vargas Al, Neblett R, Chiarotto A, et al. Dimensionality and reliability of the central sensitization inventory in a pooled multicountry sample. J Pain 2018;19:317-29.

52. Lemmens RJ, Janssen-Potten YJ, Timmermans AA, et al. Recognizing complex upper extremity activities using body worn sensors. PLoS One 2015;10:e0118642.

53. Timmermans AA, Verbunt JA, van Woerden R, et al. Effect of mental practice on the improvement of function and daily activity performance of the upper extremity in patients with subacute stroke: a randomized clinical trial. J Am Med Dir Assoc 2013;14:204-12.

54. Sullivan MJL, Bishop SR, Pivik J. The pain catastrophizing scale: development and validation. Psychol Assess 1995;7:524-32.

55. Lovibond S, Lovibond P. Manual for the depression anxiety stress scales. Sydney: School of Psychology, University of New South Wales, 1995.

56. De Vrieze T, Coeck D, Verbelen $\mathrm{H}$, et al. Cross-cultural psychometric evaluation of the Dutch McGill-QoL questionnaire for breast cancer patients. Facts Views Vis Obgyn 2016;8:205-9.

57. Brouwer S, Franche RL, Hogg-Johnson S, et al. Return-to-work self-efficacy: development and validation of a scale in claimants with musculoskeletal disorders. J Occup Rehabil 2011;21:244-58. 